\title{
DIRECT VALIDATION TECHNIQUE FOR NUMERICAL SIMULATIONS OF TIME-DEPENDENT HAMILTONIAN SYSTEMS
}

\author{
J. Struckmeier*, C. Riedel, GSI, Darmstadt, Germany
}

\section{Abstract}

Computer simulations have become an indispensable tool for studies of the physics of dynamical systems. To achieve an appropriate level of confidence in the simulation results, accuracy assessments must be conceived as an integral part of the simulation strategies. A common indirect validation method is to cross-check the results of commensurable simulation codes against each other. Furthermore, the simulation results of particularly chosen scenarios may be compared against analytical models.

In this article, we present a "direct" technique for the error assessment of numerical simulations of time-dependent Hamiltonian systems. The method is based on an invariant $I$ that has been shown to exist for $n$-degree-of-freedom Hamiltonian systems with general time-dependent potentials $[1,2]$. Because of the generally limited accuracy of numerical methods, this invariant $I$ can never be realized strictly in numerical simulations. The relative deviation of a numerically calculated "invariant" $I(t)$ from the exact invariant $I_{0}=I(0)$ may then be taken as the error estimation for the respective simulation. In this sense, the "direct" error assessment technique can be regarded as a generalization of a validation method that is applicable for autonomous (time-independent) Hamiltonian systems. For these cases, the Hamiltonian $H$ itself represents an invariant. Accordingly, the relative deviation of $H(t)$ from $H_{0}$ in the simulation is commonly used as an accuracy criterion.

Our approach is applied to estimate the accuracy of a simulation of a three-dimensional system of Coulombinteracting particles that are confined within a time-dependent quadratic external potential.

\section{INVARIANT FOR TIME-DEPENDENT HAMILTONIAN SYSTEMS}

We consider an ensemble of $N$ non-relativistic particles of the same species confined within an explicitly timedependent potential $V$. Its Hamiltonian $H$ takes the form

$$
H=\sum_{i=1}^{N} \frac{1}{2}\left[p_{x, i}^{2}+p_{y, i}^{2}+p_{z, i}^{2}\right]+V(\vec{x}, \vec{y}, \vec{z}, t) .
$$

Herein, $\vec{x}, \vec{y}$, and $\vec{z}$ denote the $N$ component vectors of the spatial coordinates of all particles; the $p_{x, y, z ; i}$ the respective canonical momenta. From the canonical equations, we derive for each particle the equation of motion

$$
\ddot{x}_{i}+\frac{\partial V(\vec{x}, \vec{y}, \vec{z}, t)}{\partial x_{i}}=0, \quad i=1, \ldots, N .
$$

\footnotetext{
*j.struckmeier@gsi.de
}

The corresponding equations hold for the $y$ - and $z$-directions. A function of all phase-space variables and time $t$

$$
I=I\left(\vec{x}(t), \vec{p}_{x}(t), \vec{y}(t), \vec{p}_{y}(t), \vec{z}(t), \vec{p}_{z}(t), t\right)
$$

is referred to as a constant of motion or invariant if and only if the value of $I$ remains constant as the system evolves in time. In other words, $d I / d t=0$ must hold along the phasespace path $\left\{\vec{x}(t), \vec{p}_{x}(t), \vec{y}(t), \vec{p}_{y}(t), \vec{z}(t), \vec{p}_{z}(t)\right\}$ defined by the system's time evolution. This path is defined as the one-parameter subset of the $6 \mathrm{~N}$-dimensional phase-space on which the equations of motion (2) are fulfilled.

In explicit form, the invariant (3) for the Hamiltonian (1) is given by $[1,2]$

$$
\begin{aligned}
I=\xi(t) H & -\frac{1}{2} \dot{\xi}(t) \sum_{i=1}^{N}\left[x_{i} p_{x, i}+y_{i} p_{y, i}+z_{i} p_{z, i}\right] \\
& +\frac{1}{4} \ddot{\xi}(t) \sum_{i=1}^{N}\left[x_{i}^{2}+y_{i}^{2}+z_{i}^{2}\right]
\end{aligned}
$$

with the function of time $\xi(t)$ representing a solution of the linear homogeneous third-order auxiliary equation

$$
\begin{aligned}
& 4 \dot{\xi}(t)\left(V+\frac{1}{2} \sum_{i=1}^{N}\left[x_{i} \frac{\partial V}{\partial x_{i}}+y_{i} \frac{\partial V}{\partial y_{i}}+z_{i} \frac{\partial V}{\partial z_{i}}\right]\right) \\
+ & 4 \xi(t) \frac{\partial V}{\partial t}+\dddot{\xi}(t) \sum_{i=1}^{N}\left[x_{i}^{2}+y_{i}^{2}+z_{i}^{2}\right]=0 .
\end{aligned}
$$

We observe that for $\partial V / \partial t \equiv 0$, i.e. for autonomous systems, $\xi(t) \equiv 1$ is always a solution of Eq. (5). For this $\xi(t)$, the invariant (4) reduces to $I \equiv H$, hence coincides with the Hamiltonian $H$ as a well-known invariant for systems with no explicit time-dependence.

As the coefficients of the auxiliary equation (5) generally depend on all spatial coordinates, the integral function $\xi(t)$ can only be calculated in conjunction with the solution of the equations of motion (2). With the known particle trajectories, all coefficients of Eq. (5) are in fact functions of the parameter time only. Then, Eq. (5) represents a linear ordinary differential equation for $\xi(t)$ with time-dependent coefficients. According to the existence and uniqueness theorem for linear ordinary differential equations, a unique solution $\xi(t)$ of Eq. (5) exists - and consequently the invariant $I$ of Eq. (4) - if the given potential function $V$ and its partial derivatives are continuous.

With $\xi(t)$ a solution of (5), we may directly prove that $d I / d t=0$ holds along the phase-space path defined by the solution of the equations of motion (2). Substituting 
Eqs. (2) into the total time derivative of (4), we find that the surviving terms agree with the auxiliary equation (5). Hence, Eq. (4) provides a conserved quantity $I$ as a time integral of Eq. (5) if and only if the system's evolution is governed by the equations of motion (2). We will use this relationship in Sec. 3 to estimate the numerical error of computer simulations of a system of Coulomb-interacting particles. Beforehand, we work out the invariant and the auxiliary equation in the particular form pertaining to this Hamiltonian system.

\section{INVARIANT FOR A SYSTEM OF COULOMB-INTERACTING PARTICLES}

We now consider an ensemble of $N$ Coulomb-interacting particles of the same species moving in a timedependent quadratic external potential, as given in the comoving frame for charged particle beams that propagate through linear external focusing devices. The potential function $V$ of this system is given by

$$
\begin{aligned}
V(\vec{x}, \vec{y}, \vec{z}, t)=\sum_{i=1}^{N} & {\left[\frac{1}{2} \omega_{x}^{2}(t) x_{i}^{2}+\frac{1}{2} \omega_{y}^{2}(t) y_{i}^{2}\right.} \\
& \left.+\frac{1}{2} \omega_{z}^{2}(t) z_{i}^{2}+\frac{1}{2} \sum_{j \neq i} \frac{c_{0}}{r_{i j}}\right],
\end{aligned}
$$

with $r_{i j}^{2}=\left(x_{i}-x_{j}\right)^{2}+\left(y_{i}-y_{j}\right)^{2}+\left(z_{i}-z_{j}\right)^{2}$ and $c_{0}=q^{2} / 4 \pi \epsilon_{0} m, q$ and $m$ denoting the particles' charge and mass, respectively. The $3 N$ equations of motion that follow from (2) with (6) are

$$
\ddot{x}_{i}+\omega_{x}^{2}(t) x_{i}-c_{0} \sum_{j \neq i} \frac{x_{i}-x_{j}}{r_{i j}^{3}}=0,
$$

and likewise for the $y$ and $z$ degrees of freedom. We note that the factor $1 / 2$ in front of the Coulomb interaction term in (6) disappears since each term occurs twice in the symmetric form of the double sum. With the potential (6), the auxiliary equation (5) specializes to

$$
\begin{aligned}
& \left\langle x^{2}\right\rangle\left(\dddot{\xi}+4 \dot{\xi} \omega_{x}^{2}+4 \xi \omega_{x} \dot{\omega}_{x}\right) \\
+ & \left\langle y^{2}\right\rangle\left(\dddot{\xi}+4 \dot{\xi} \omega_{y}^{2}+4 \xi \omega_{y} \dot{\omega}_{y}\right) \\
+ & \left\langle z^{2}\right\rangle\left(\dddot{\xi}+4 \dot{\xi} \omega_{z}^{2}+4 \xi \omega_{z} \dot{\omega}_{z}\right)+\frac{2 W(t)}{m N} \dot{\xi}=0 .
\end{aligned}
$$

Herein, the sums over the squares of the particle coordinates are written in terms of "second beam moments", denoted as $\left\langle x^{2}\right\rangle$ for the $x$-direction. Furthermore, $W(t)$ stands for the electrostatic field energy constituted by all particles

$$
\left\langle x^{2}\right\rangle(t)=\frac{1}{N} \sum_{i=1}^{N} x_{i}^{2}(t), \quad W(t)=\frac{m}{2} \sum_{i=1}^{N} \sum_{j \neq i} \frac{c_{0}}{r_{i j}} .
$$

The invariant $I$ for this system is given by (4), provided that $\xi(t)$ is a solution of the auxiliary equation (8). As already stated above, its time-dependent coefficients $\left\langle x^{2}\right\rangle(t)$, $\left\langle y^{2}\right\rangle(t),\left\langle z^{2}\right\rangle(t)$, and $W(t)$ must be known as the result of the simultaneous integration of the equations of motion (7). Again, we may directly prove that the invariant (4) with (1) and (6) constitutes a time integral of the auxiliary equation (8) by calculating the total time derivative of (4) and inserting the single particle equations of motion Eq. (7).

Defining $\omega^{2}(t)$ as the "average force function"

$$
\omega^{2}(t)=\frac{\omega_{x}^{2}\left\langle x^{2}\right\rangle+\omega_{y}^{2}\left\langle y^{2}\right\rangle+\omega_{z}^{2}\left\langle z^{2}\right\rangle}{\left\langle x^{2}\right\rangle+\left\langle y^{2}\right\rangle+\left\langle z^{2}\right\rangle},
$$

the linear third-order auxiliary equation (8) may be expressed equivalently in terms of a coupled set of a nonlinear second-order equation for $\xi(t)$

$$
\xi \ddot{\xi}-\frac{1}{2} \dot{\xi}^{2}+2 \omega^{2}(t) \xi^{2}=g(t),
$$

and a first-order equation for $g(t)$. Comparing the time derivative of (9) with (8), one finds that $\dot{g}(t)$ must satisfy

$$
\begin{gathered}
\dot{g}(t)=\frac{1}{\left\langle x^{2}\right\rangle+\left\langle y^{2}\right\rangle+\left\langle z^{2}\right\rangle}\left[-2 \xi \dot{\xi} \frac{W}{m N}+4 \xi^{2}\{\right. \\
\left.\left.\left\langle x p_{x}\right\rangle\left(\omega_{x}^{2}-\omega^{2}\right)+\left\langle y p_{y}\right\rangle\left(\omega_{y}^{2}-\omega^{2}\right)+\left\langle z p_{z}\right\rangle\left(\omega_{z}^{2}-\omega^{2}\right)\right\}\right] .
\end{gathered}
$$

Unlike the third-order auxiliary equation (8), the equivalent coupled set of equations (9) and (10) no longer contains the time derivatives of the external focusing functions $\omega_{x}^{2}(t), \omega_{y}^{2}(t)$, and $\omega_{z}^{2}(t)$. It has been shown in Ref. [1] that $\dot{g}(t) \equiv 0$ in the case of a one-dimensional linear system. $\dot{g}(t)$, as given by Eq. (10), thus quantifies the contribution of our actual three-dimensional non-linear system to the solution $\xi(t)$ of the second-order auxiliary equation (9). We learn from Eq. (10) that $\dot{g}(t)$ is determined by two quantities of different physical nature: the field energy $W$ constituted by all particles as a measure for the strength of the Coulomb interaction, and the system's anisotropy.

With the help of Eq. (9), we may substitute $\ddot{\xi}(t)$ and the external focusing functions in (4) to express the invariant $I$ in the alternative form

$$
\begin{aligned}
I=\frac{N}{2 \xi} & {\left[\left\langle\left(\xi p_{x}-\frac{1}{2} \dot{\xi} x\right)^{2}\right\rangle+\left\langle\left(\xi p_{y}-\frac{1}{2} \dot{\xi} y\right)^{2}\right\rangle\right.} \\
& +\left\langle\left(\xi p_{z}-\frac{1}{2} \dot{\xi} z\right)^{2}\right\rangle+\xi^{2} \frac{2 W}{m N} \\
& \left.+\frac{1}{2} g(t)\left(\left\langle x^{2}\right\rangle+\left\langle y^{2}\right\rangle+\left\langle z^{2}\right\rangle\right)\right] .
\end{aligned}
$$

In the following section, we will numerically calculate the invariant (11) as a function of time in order to estimate the accuracy of a simulation of a system governed by the coupled set of $3 N$ second-order equations (7).

\section{CHECKING SIMULATIONS OF COULOMB-INTERACTING PARTICLES}

In the ideal case, i.e. if no numerical inaccuracies were included in a computer simulation of a Hamiltonian system, and no numerical errors were added performing the 
subsequent integration of the auxiliary equation, we would not experience any deviation $\Delta I=I(t)-I_{0}$ calculating the invariant $I(t)$ as a function of time.

Of course, we can never avoid numerical errors to occur in computer simulations of dynamical systems because of the generally limited accuracy of numerical methods. For the same reason, the numerical integration of the auxiliary equation is also associated with a specific finite error tolerance. Therefore, the numerically calculated value of $I$ as given by Eq. (11) - with $\xi(t), \dot{\xi}(t)$, and $g(t)$ following from (9) and (10) - can never be exactly constant. Both numerical problems, the numerical integration of the equations of motion (7), and the subsequent numerical integration of the coupled set of Eqs. (9), (10) contribute to a nonvanishing $\Delta I / I_{0}$ along the integration time span. Since both problems do not depend on each other with respect to their specific error tolerances, we may regard the resulting $\Delta I / I_{0}$-curve as a cross-check of both numerical methods.

We can directly estimate from $\Delta I(t) / I_{0}$ the error tolerance integrating the $6 \mathrm{~N}$ first-order equations of motion corresponding to (7) if the error from the numerical integration of the three first-order equations that correspond to Eqs. (9) and (10) can be neglected. This can be achieved by setting the time step size together with the error tolerances for the integration of the auxiliary equation appropriately.

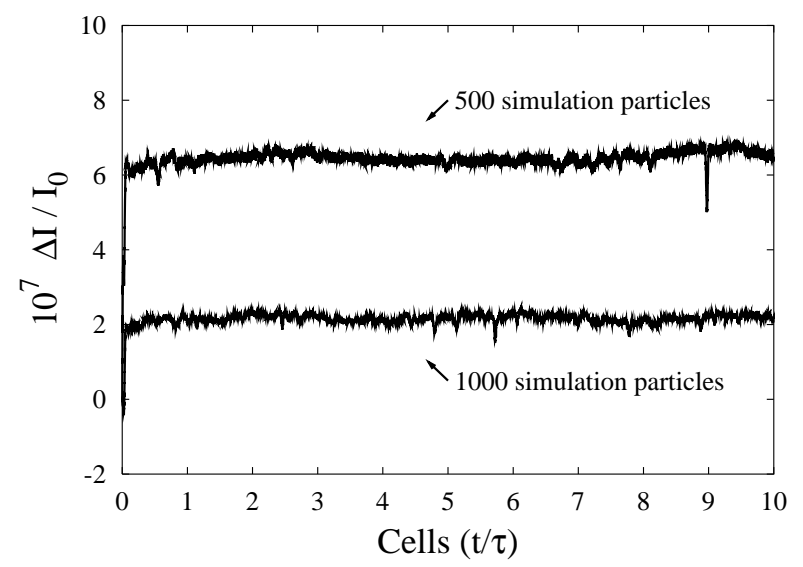

Figure 1: Relative invariant errors $\Delta I / I_{0}$ for three-dimensional simulations of a charged particle beam with different numbers of macro-particles and only the "natural" error of the numerical calculation. $\tau$ denotes the length of the focusing period.

Fig. 1 displays two examples of curves of relative deviations $\Delta I / I_{0}$ from the invariant (11) for numerical simulations of a charged particle beam. The functions $\xi(t)$, $\dot{\xi}(t)$, and $g(t)$ that are needed to calculate $I$ were obtained from a numerical integration of the coupled set (9) and (10). The time-dependent coefficients, namely the second beam moments and the field energy $W(t)$ had been determined beforehand from three-dimensional simulations of charged particle beams propagating through a linear focusing lattice with non-negligible Coulomb interaction, as described by the potential function (6). As expected, the residual devia- tion $\Delta I / I_{0}$ depends on the number of macro-particles used in the simulation.

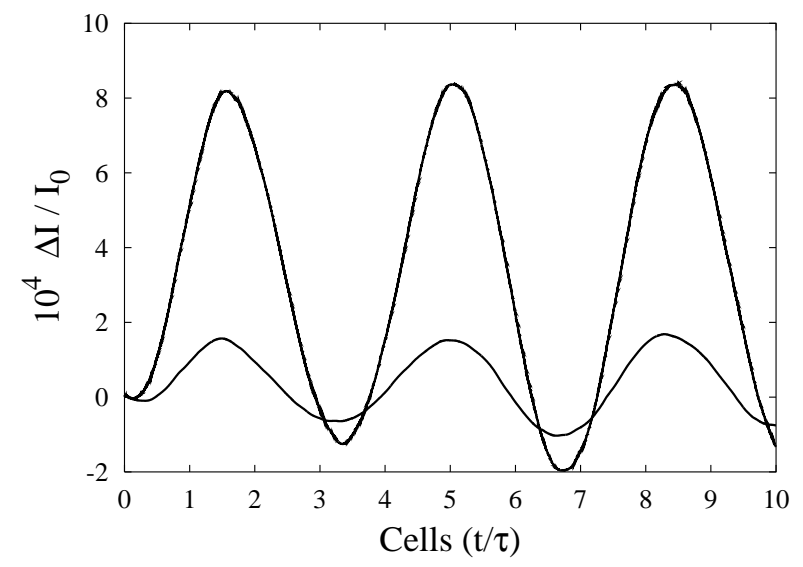

Figure 2: Relative invariant errors $\Delta I / I_{0}$ for a three-dimensional simulation of a charged particle beam with systematic $2.5 \%$ - and $5 \%$-errors in the space-charge force calculations. $\tau$ denotes the length of the focusing period.

For a comparison, the corresponding deviations are plotted in Fig. 2 for the simulation of Fig. 1, but now comprising systematic $2.5 \%$ - and $5 \%$-errors in the space-charge force calculations. We now find macroscopic oscillations of the relative invariant deviation $\Delta I / I_{0}$ in the order of $10^{-3}$, hence three orders of magnitude larger than the corresponding simulation with no systematic space-charge force error. We conclude that the "direct validation technique" may also serve the purpose to pinpoint eventual systematic errors in a simulation algorithm.

\section{CONCLUSIONS}

The existence of a constant of motion $I$ for general explicitly time-dependent Hamiltonian systems has been shown to be useful to check the accuracy of numerical simulations of this class of dynamical systems. Having numerically integrated the equations of motion, the system's auxiliary function $\xi(t)$ can be numerically calculated, and the numerical value of the "invariant" $I(t)$ be obtained thereof. The relative deviation $\Delta I / I_{0}$ of $I(t)$ from the exact invariant $I_{0}$ - defined by the initial conditions - can then be used as a measure for the accuracy of the respective simulation. Comparing simulation runs with different parameters, such as the number of macro-particles, the time step size, details of the numerical algorithm used to integrate the equations of motion, we may straightforwardly check whether the overall accuracy of our particular simulation has been improved.

\section{REFERENCES}

[1] J. Struckmeier and C. Riedel, Phys. Rev. Lett. 85, 3830 (2000)

[2] J. Struckmeier and C. Riedel, Phys. Rev. E 63, (2001) (to be published) [accepted for publication] 